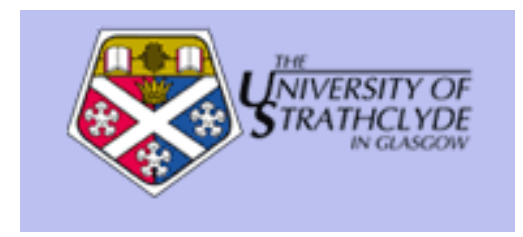

Bradley, N.A. and Dunlop, M.D. (2005) Towards a multidisciplinary model of 'context' to support context-aware computing. Journal of Human-Computer Interaction, 20 (4). pp. 403-446. ISSN 1745-4360

http://eprints.cdlr.strath.ac.uk/2602/

This is an author-produced version of a paper published in The Journal of Human-Computer Interaction, ISSN 1745-4360.

This version has been peer-reviewed, but does not include the final publisher proof corrections, published layout, or pagination.

Strathprints is designed to allow users to access the research output of the University of Strathclyde. Copyright (c) and Moral Rights for the papers on this site are retained by the individual authors and/or other copyright owners. Users may download and/or print one copy of any article(s) in Strathprints to facilitate their private study or for non-commercial research. You may not engage in further distribution of the material or use it for any profitmaking activities or any commercial gain. You may freely distribute the url (http://eprints.collr.strath.ac.uk) of the Strathprints website.

Any correspondence concerning this service should be sent to The Strathprints Administrator: eprints@cis.strath.ac.uk 


\title{
Towards a Multidisciplinary Model of Context to Support Context-Aware Computing
}

\author{
Nicholas A. Bradley and Mark D. Dunlop \\ Strathclyde University
}

\begin{abstract}
Capturing, defining, and modeling the essence of context are challenging, compelling, and prominent issues for interdisciplinary research and discussion. The roots of its emergence lie in the inconsistencies and ambivalent definitions across and within different research specializations (e.g., philosophy, psychology, pragmatics, linguistics, computer science, and artificial intelligence). Within the area of computer science, the advent of mobile context-aware computing has stimulated broad and contrasting interpretations due to the shift from traditional static desktop computing to heterogeneous mobile environments. This transition poses many challenging, complex, and largely unanswered research issues relating to contextual interactions and usability. To address those issues, many researchers strongly encourage a multidisciplinary approach. The primary aim of this article is to review and unify theories of context within linguistics, computer science, and psychology. Summary models within each discipline are used to propose an outline and detailed multidisciplinary model of context involving (a) the

Nicholas A. Bradley is an HCI researcher with an interest in the usability of context-aware systems; he is a $\mathrm{PhD}$ student in the Department of Computer and Information Sciences, Strathclyde University, Glasgow. Mark D. Dunlop is computer scientist with an interest in the usability and evaluation of mobile systems, context-aware systems, and novel text input methods; he is a Senior Lecturer in the Department of Computer and Information Sciences, Strathclyde University, Glasgow.
\end{abstract}




\section{Contents}

\section{INTRODUCTION}

2. DEFINITIONS AND CATEGORIZATIONS OF CONTEXT

2.1. General Definitions of Context

2.2. Linguistics and Context Usability Issues for Context-Aware Applications

Proposed Summary Propeller Model of Linguistic Context

2.3. Computer Science and Context

Definitions of Context

Categorizations of Context

Models of Context

Contrasting Definitions, Categorizations, and Models

Proposed Summary Model of Context in Computer Science

2.4. Psychology and Context

Usability Issues for Context-Aware Applications

Proposed Summary Model of Context in Psychology

2.5. Context Within Other Research Areas

3. CONTRASTING AND ANALYZING PROPOSED MODELS

3.1. Contextual Interactions

3.2. The Notion of Relevancy

3.3. Proposed Multidisciplinary Definition of Context

4. PROPOSED MULTIDISCIPLINARY MODEL OF CONTEXT

4.1. Differentiation of User and Application'S World

4.2. Separation Between Meaningful and Incidental Dimensions

Quadrant 1

Quadrant 2

Quadrant 3

Quadrant 4

4.3. Application and User Processes

User Processes

Application Processes

5. APPLYING THE MULTIDISCIPLINARY MODEL TO DIFFERENT APPLICATIONS OF CONTEXT-AWARE COMPUTING

5.1. Mobile Tourist Guides

5.2. Mobile User Communities

6. CONCLUSION

5.3. Applying the Model in Practice

7. DISCUSSION

differentiation of focal and contextual aspects of the user and application's world, (b) the separation of meaningful and incidental dimensions, and (c) important user and application processes. The models provide an important foundation in which complex mobile scenarios can be conceptualized and key human and social issues can be identified. The models were then applied to different applica- 
tions of context-aware computing involving user communities and mobile tourist guides. The authors' future work involves developing a user-centered multidisciplinary design framework (based on their proposed models). This will be used to design a large-scale user study investigating the usability issues of a context-aware mobile computing navigation aid for visually impaired people.

\section{INTRODUCTION}

The phenomenon of context has become an increasingly intriguing multidisciplinary talking point. Davies and Thomson (1988) remarked that the main reason for context assuming a central role in various research areas is "the acknowledgement, explicit or implicit, that organisms, objects and events are integral parts of the environment and cannot be understood in isolation of that environment." Although context is frequently cited, and its importance repeatedly proclaimed, Dervin (1997) argued that it is rarely given a detailed philosophical and theoretical treatment, in particular to its multiple interdependencies; its dialectical relationships between product and process; and its temporal and spatial (i.e., here-and-now) confluence of people, settings, activities, and events.

The roots of its emergence also lie in the inconsistencies and ambivalent definitions across and within different research specializations (e.g., philosophy, psychology, pragmatics, linguistics, and artificial intelligence). Benerecetti, Bouquet, and Ghidini (2001) stipulated that a general and unifying theory or formalization of context is still in its infancy and that it is unclear whether each research area is addressing aspects of the same problem or different problems with the same name.

Chen and Kotz (2000) illustrated the discrepancies in the use of the word context within different areas of computer science (e.g., context-sensitive help, contextual search, multitasking context switch, etc.). The advent of mobile context-aware computing, for instance, has stimulated broad and contrasting interpretations, due to the shift from traditional static desktop computing to heterogeneous mobile environments. This transition poses many challenging, complex, and largely unanswered research issues relating to contextual interactions and usability.

For context-aware systems to seamlessly support and enrich a user's mobile activities, there is a need to understand context from a multidisciplinary viewpoint (Bradley \& Dunlop, 2003; Mynatt, Essa, \& Rogers, 2000; Selker \& Burleson, 2000). Current design frameworks, however, are predominantly software oriented (e.g., Huang, 2002; Kim, Yae, \& Ramakrisha, 2001), making it difficult to capture and effectively manage human variability. Bellotti 
and Edwards (2001) stated that "it is the human and social aspects of context which are crucial in making a context-aware system a benefit rather than a hindrance - or even worse - an annoyance." Selker and Burleson (2000) stipulated how context-aware design needs to explicitly draw on cognitive science, user experience, and situation in the design process.

Many researchers in computer science have illustrated the benefits of understanding context. Dey (2001) remarked that it could lead to improved usability of context-aware applications. Dey and Abowd (1999) stated that "by improving the computer's access to context, we increase the richness of communication in Human-Computer Interaction (HCI), making it possible to produce more useful computational services." An understanding of context will also enable application designers to choose what context to use in their applications, therefore helping them to determine what context-aware behaviors to support. Brezillon and Abu-Hakima (1995) remarked that context plays an important role in person-machine and machine-machine interactions and in the representation of knowledge-based systems.

Context is also analyzed and discussed considerably within industry and is regarded as the key to unlocking the true value of business applications on handheld devices (Zetie, 2002a). For example, Sun Microsystems, Giga Information Group, and US Bancorp Piper Jaffray have recently identified context as a key enabling technology missing from today's mobile platform applications (Unwired Express, 2002). It is therefore not surprising that next-generation applications and Web services are increasingly taking into account the user's context to use contextual information to modify the application's business logic, presentation, and navigation.

The primary aim of this article is to review and merge theories of context within linguistics, computer science, and psychology, to propose a multidisciplinary model of context that would facilitate application developers in developing richer descriptions or scenarios of how a context-aware device may be used in various dynamic mobile settings. More specifically, the aim is to:

1. Investigate different viewpoints of context within linguistics, computer science, and psychology, to develop summary condensed models for each discipline.

2. Investigate the impact of contrasting viewpoints on the usability of context-aware applications.

3. Investigate the extent to which single-discipline models can be merged and the benefits and insightfulness of a merged model for designing mobile computers.

4. Investigate the extent to which a proposed multidisciplinary model can be applied to specific applications of context-aware computing. 
We anticipate that this review will provide a stepping stone to the development of a user-centered and multidisciplinary design framework for building and assessing context-aware applications. The purpose of this article is not to advance the philosophical debates on context, such as the phenomenological or positivist accounts of action (which are widely covered elsewhere, e.g., Dervin, 1997) but to draw on theories of context within each discipline to illustrate their practical implications for designing mobile context-aware computers. This article is therefore intended for application developers, although more exclusively for usability researchers (advancing user-centered design theories, models, or frameworks) and usability practitioners (incorporating key design and usability context principles in an applied setting).

\section{DEFINITIONS AND CATEGORIZATIONS OF CONTEXT}

This section is divided into the following key areas: (a) general definitions of context, (b) linguistics and context, (c) computer science and context, (d) psychology and context, and (e) context within other research areas. The investigation focuses on the disciplines of linguistics, computer science, and psychology because their theories and principles were considered to be the most applicable to, and beneficial for, context-aware computing.

Definitions and categorizations of context are provided within Sections 2.1 through 2.5. Within Sections $2 \mathrm{~b}$ through $2 \mathrm{~d}$, we present a proposed model of context and a subjective analysis in relation to context-aware computing. The purpose of creating models was to (a) show conceptually how context is interpreted within each discipline, (b) facilitate the visual and theoretical identification of similarities and links for creating a multidisciplinary model, and (c) illustrate how principles within other disciplines have direct implications for context-aware computing.

\subsection{General Definitions of Context}

The Chambers 21st Century Dictionary (1996) and the Oxford Pocket Dictionary of Current English (1992) both define context as either "the pieces of writing in a passage which surround a particular word, phrase, paragraph, and so on," and "the circumstances, background or setting." Benerecetti et al. (2001) provided a more analytical perspective of context. As described in Figure 1, three dimensions of context dependence ${ }^{1}$ are identified: (a) partiality, (b) approximation, and (c) perspective.

1. Context dependenceimplies that when some aspect of context is used explicitly or intrinsically in a given situation, that aspect of context is required for that situation to occur. 
Figure 1. Dimensions of Context Dependence.

\begin{tabular}{|c|c|}
\hline Dimension & Definition \\
\hline Partiality & $\begin{array}{l}\text { A context-dependent representation is partial when it describes only a } \\
\text { subset of a more comprehensive state of affairs. There are two } \\
\text { perspectives: (a) metaphysical - a representation is partial if it does not } \\
\text { cover the entire universe and (b) cognitive - a representation is partial if } \\
\text { it does not include the entirety of what a person can talk about. }\end{array}$ \\
\hline Approximation & $\begin{array}{l}\text { A context-dependent representation is approximate when it abstracts } \\
\text { away some aspects of a given state of affairs. The aspects abstracted } \\
\text { away are taken into account in some other form of representation. }\end{array}$ \\
\hline Perspective & $\begin{array}{l}\text { A representation is perspectival when it encodes a spatiotemporal, } \\
\text { logical, or cognitive point of view on a state of affairs. For example, } \\
\text { the statement "It's snowing" implies a spatial perspective (i.e., the } \\
\text { location in which the statement is used) and a temporal perspective } \\
\text { (i.e., it is snowing now). Additionally, some statements, such as "hot air } \\
\text { rises," imply a logical perspective as they implicitly refer to this world. }\end{array}$ \\
\hline
\end{tabular}

In relation to context-aware computing, the approximation dimension is closely tied to the notion of relevancy (discussed in Section 3.4). Contextual information not required by the user must be abstracted away, making the remaining information partial. The perspective dimension, however, indicates how information must account for a spatial perspective (e.g., a user's location), temporal perspective (e.g., is the information relating to the past, present, or future?), and logical perspective (e.g., does the information match that of the surrounding environmental context?). Although these context-dependence principles stimulate ruminative discussion, it is unclear how they can be used to develop techniques for capturing, measuring, and assessing parameters of context within complex tasks and systems.

\subsection{Linguistics and Context}

Researchers in the area of linguistics and communication have studied many aspects of context. These include: (a) the changes in utterance interpretation when spoken in different contexts, (b) the production of a speaker's utterance in accordance with what he or she perceives is the current conversational context, and (c) the method in which a hearer selects or constructs the context in order to comprehend the message.

Fetzer (1997) defined context as a "tripartite system of objective, social and subjective worlds, their sub-systems and presuppositions." The objective world is measurable and consists of a true-false paradigm. The subjective world is characterized by sincerity in that a speaker's conversational intention is spoken as intended and the social world is represented by textual, interper- 
sonal, and interactional meaning. In other viewpoints, Ochs (1979) distinguished between the social and psychological worlds. These include people's beliefs and assumptions about (a) temporal, spatial, and social settings; (b) prior, ongoing, and future actions (both verbal and nonverbal); and (c) the state of knowledge and attentiveness of those participating in the social interaction.

Categorizations of contextual information used within a communication act have been proposed. Bunt (1997) believes that the relevant factors of conversational context can be grouped into five categories:

1. Linguistic: Properties of the surrounding linguistic material (textual or spoken).

2. Semantic: Constructed by the underlying task and the task domain (the objects, properties, and relations relevant to the task).

3. Physical: The physical circumstances/environment in which the interaction occurs.

4. Social: The type of interactive situation, combined with the participant's roles in that situation, as depicted in terms of their communicative rights and obligations.

5. Cognitive: The participants' beliefs, intentions, plans and other attitudes; their states of processing relating to perception, production, interpretation, evaluation, execution; and their attentional states.

Connolly (2001) separated between the linguistic context and nonlinguistic (or situational) context. Linguistic context refers to the units that give meaning to words, phrases, or sentences. Connolly identified two types:

1. Cotext: The text that surrounds a unit of language (words, phrases, etc.) that gives its linguistic context. This is similar to the concept of anaphora, whereby the coreference of one expression is made with its antecedent. The antecedent provides the information necessary for the expression's interpretation - for example, when the name David is replaced with his in for succeeding text.

2. Intertext (or intertextuality): The notion that, to comprehend/interpret part of one text, information from some other text may be required. Situational context refers to the pertinent aspects of the environment that are nontextual in nature (but where the text exists), for example, an author's cognitive decisions and ideas regarding the compilation of the text.

Researchers in linguistics have also investigated how people behave and interact with context. A theory of meaning and communication, called situation theory, is used to depict various types of situation (Connolly, 2001). In one representation, a hierarchical formation is used to illustrate the utterance situa- 
tion (at the bottom of hierarchy), which consists of who is addressing whom, where, and when, and what utterances are produced. The discourse situation relates to the entire conversation, which is part of the embedding situation (an accumulation of discourses). At the top of the hierarchy, the world depicts the maximal situation in which all other situations occur. The meaning of language was also addressed extensively by Wittgenstein (1958), who believed that the meaning of sentences depends on the context of utterance. It is described how words do denote meaning in isolation, but only when used inside a language game consisting of a social environment of speech and action. The term language game is used to illustrate that speaking of language is part of an activity. So if the sentence, for instance, is the basic move in the language game, a language game itself is taken to be the basic unit in linguistic activity. Words become meaningful only when we consider the occasion on and purpose for which it is said.

In other investigations, Bunt (1997) described how human dialogue consists of two simultaneous tasks: (a) attempting to achieve the underlying noncommunicative goal and (b) communicating to achieve the associated communicative goal. To illustrate, if a person is visiting a car dealership with the intent of purchasing a car, the noncommunicative goal (which will motivate a dialogue) may be to buy a car within a particular price range. However, although the communicative goal will be to verbalize this to the car salesman, the noncommunicative goal will be reshaped as the salesman's feedback is being weighed and compared with the original noncommunicative goal (thereby allowing the salesman to convince the buyer that this more expensive car is justified!).

Last, Fetzer (1997) illustrated how speakers/hearers create and interpret their utterances in and through an already-existing context. Therefore, speakers link and anchor their utterances to that context. Fetzer also pointed out that context represents both a process and a result as it is selected and constructed through an act of verbal and nonverbal communication. It is selected in the sense that the speaker selects contextual information on the basis of previous communication acts and then accepts or rejects this information and it is constructed in the sense that the speaker adds new contextual information to the already-existing contextual information through his or her response.

\section{Usability Issues for Context-Aware Applications}

Bunt's (1997) research can be used to model the interaction of a user with a context-aware application, as illustrated in Figure 2.

The upper oval circle in Figure 2 represents the user, the lower oval shape depicts the context-aware application, and the area surrounding the oval circles illustrates the environment. To illustrate the key principles of Figure 2, we 
Figure 2. Modeling user and application communicative and noncommunicative goals.

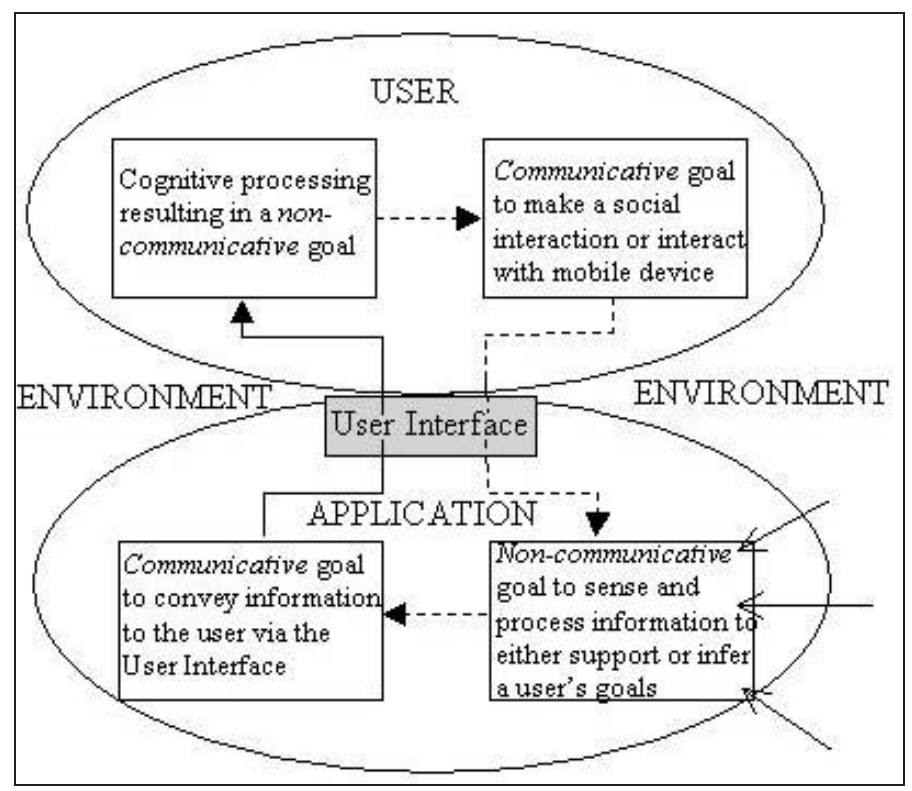

use a scenario involving a person, Alice, who is traveling to the train station using her mobile computing travel aid.

Alice makes a noncommunicative cognitive goal to travel to the train station to catch her last train home (i.e., a goal formed without communication with the mobile device). She may need to construct a communicative goal, or an interactional goal, in which to solicit trip information (e.g., which actions are required to obtain travel directions via the user interface?). This would result in a noncommunicative application goal to sense and process information in which to support her query/instruction. However, before Alice realizes her communicative goal, the context-aware application may be able to infer her noncommunicative goal from sensed information, such as the time of day and GPS location (thereby making the user's communicative goal unnecessary). In either situation, the application would need to execute a communicative goal to transmit travel details appropriately, comprehensibly, and in a timely manner with respect to Alice's personal preferences, situation, and environment. Other noncommunicative application goals may be to actively check the status of the train, which is communicated to her only if a delay is encountered. As a result, this information would be given to Alice, who would cognitively process it and then possibly form additional noncommunicative goals (e.g., stop at nearest café for a drink). 
The preceding scenario, in relation to Fetzer's (1997) research, also illustrates how a context-aware application must link and anchor information in accordance to the user's current context, as described next:

- Selected application intelligibility: To make accurate inferred decisions regarding Alice's current and future intentions, the application may need to select previously captured information regarding her behavioral patterns. The application, for instance, may have tracked from previous train and bus delays that Alice visits cafés and bookshops to pass time; subsequently, the application could automatically select, or make informed, recommendations.

- Constructed application intelligibility: This is temporally driven as the application contributes to constructing present and future user contexts. The application must therefore intelligently filter, modify, or add to information from past contexts to suitably construct Alice's current context. For instance, in the preceding scenario, the application creates a context by informing her that the train is delayed. She is now aware of this and may not require this information again unless the status of the train changes. A key issue would be: To what extent should the application construct Alice's context-how much should be left to her own interpretation? If Alice is about to walk past a café after being told of a train delay, should the application assume she has spotted this or should it provide an inferred recommendation regardless?

To illustrate this last point further, with respect to Connolly's (2001) description of intertextuality and cotext, the application must be sensitive to Alice's acquired knowledge and experience. The application, for instance, may not need to alert Alice of train departure times if she has already acquired this knowledge. In terms of intertextuality, she will be referring more to knowledge-based information than to application-based information. However, to what extent can assumptions be made relating to user knowledge, experience, and memory capabilities when considering the diversity of human capabilities (and disabilities)?

\section{Proposed Summary Propeller Model of Linguistic Context}

These viewpoints of linguistic context have been captured and used to create our summary propeller model of context, as illustrated in Figure 3.

The model represents how, prior to an utterance, a person, represented by either Cognitive Context 1 or 2, first processes (i.e., rejects or accepts contextual information) and selects a noncommunicative goal, which is then used to select a communicative goal. The communicative goal of verbalizing the asso- 
Figure 3. Our proposed propeller model of context within the linguistics domain. Com. $=$ communicative; Non-com. $=$ noncommunicative.

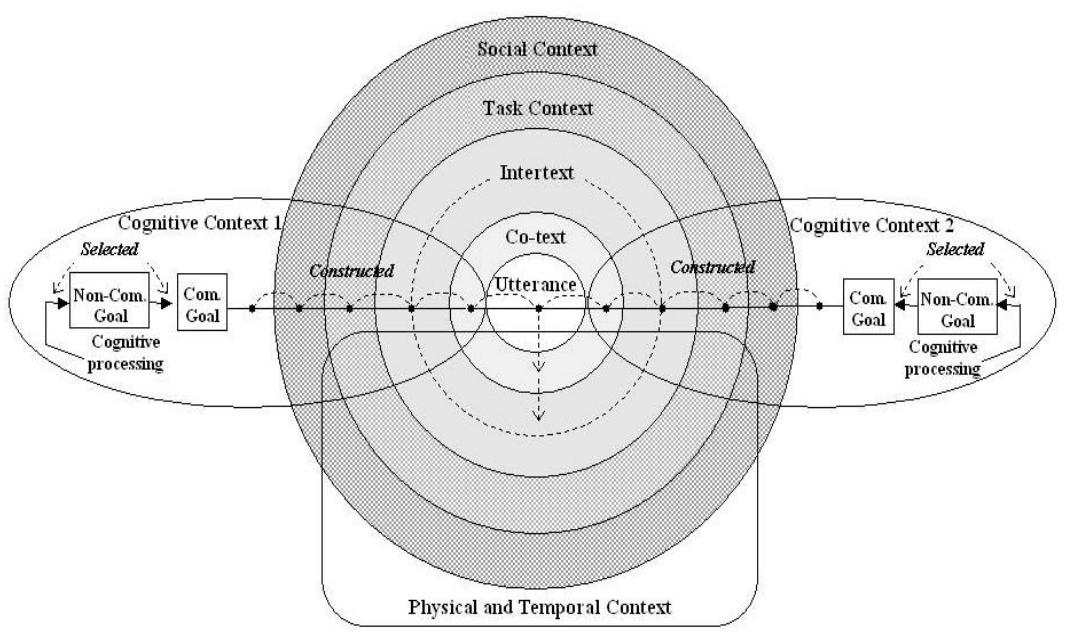

ciated noncommunicative goal then shapes/constructs the social and task context, the intertext, and the cotext. Whether it is an utterance or discourse, both the cognitive contexts will expand with more contextual information, which is used to process and select future goals.

After the utterance has occurred, this becomes the cotext to the next utterance. Similarly, after this discourse, the utterance will form the intertext to the next discourse. Last, all of this operates within a physical and temporal context (i.e., it has been influenced by previously occurring events).

\subsection{Computer Science and Context}

The notion of context is a powerful and long-standing concept in human-computer interaction. Over the years, computer scientists have contributed to an expanding and varied list of definitions, categorizations, and models, examples of which we now describe and contrast and use to propose a model of context for computer science.

\section{Definitions of Context}

Computer scientists' definitions of context can be loosely placed into three distinct types: those that describe the primary focus of context from the perspective of (a) the application, (b) the user, and (c) any entity of interest. 
Primary Focus as the Application. Moran and Dourish (2001) defined context as the "physical and social situation in which computational devices are embedded." Ward, Jones, and Hopper (1997) view context as the state of the application's surroundings. Similarly, Brown (1996) defined context as the elements of the user's environment that the user's computer knows about. Chen and Kotz (2000) defined context as the "set of environmental states and settings that either determines an application's behaviour or in which an application event occurs and is interesting to the user."

Primary Focus as the User. Dey, Abowd, and Wood (1999) defined context as the user's physical, social, emotional, or informational state, whereas Zetie (2002b) described context in software applications as "the knowledge about the goals, tasks, intentions, history and preferences of the user that a software application applies to optimizing the effectiveness of the application." Zetie (2002b) also identified the key dimensions of context by asking questions about the user: who the user is (e.g., personal characteristics), what the user is doing (i.e., activity/task), where the user is, and how to contact the user (based on his or her location, time of day, preferences, priority of interaction, etc.). Similarly, Schilit, Adams, and Want (1994) treated the user as the primary entity to ask questions such as where you are, who you are with, and what resources are nearby.

Primary Focus as Any Entity of Interest. Schmidt (2001) views context as facts that matter for an application/user/device, which are inherently connected to time and location. In more general definitions, Funk and Miller (1997) described context as "everything surrounding an item of interest, including the 'mindset' of any humans involved in the context." Similarly, Dey and Abowd (1999) defined context as "any information that can be used to characterize the situation of an entity. An entity is a person, place, or object that is considered relevant to the interaction between the user and an application, including the user and application themselves."

\section{Categorizations of Context}

A variety of categorizations of context have also been proposed. Common categories include a user's location and environment, identities of nearby people and objects, and changes to those objects (Brown, Bovey, \& Chen, 1997; Dey, 1998; Ryan, Pascoe, \& Morse, 1997; Schilit \& Theimer, 1994), whereas some use additional categories such as time of day (Brown et al., 1997; Ryan et al., 1997) and the user's emotional state and focus of attention (Dey, 1998).

Schilit and Thiemer (1994) differentiated among three broad types of context: (a) the computing environment, which includes available processors, de- 
vices accessible for user input and display, network capacity, connectivity, and computing costs; (b) the user environment, which depicts the location, collections of nearby people, and social situation; and (c) the physical environment, which includes parameters such as lighting and noise level. Chen and Kotz (2000), however, stipulated that this categorization neglects the time context. Temporal recordings of user, computer, and physical contexts provide useful sources for supporting applications.

\section{Models of Context}

Coutaz and Rey (2002) proposed a mathematical model in which the context of a situation is defined as the "set of periphic variables and relations between them." The following formula represents how a series of situations (i.e., snapshots in time) are combined to give the context for a user $(U)$, task $(T)$, and time $(t)$ :

\section{Context $(U, T, t)=\operatorname{Cumul}\left[\operatorname{Situation}\left(U, T, t_{0}\right) \ldots\right.$ Situation $\left.(U, T, t)\right]$}

In this formula, Cumul is a union operation that labels periphic variables and remaps relationships between variables as necessary. Coutaz and Rey also introduce the notion of contextor, which is a reflexive and hierarchically composable context sensor with data inputs and outputs plus control inputs and outputs.

In other models of context, Zetie (2002b) illustrated the interactions between an application and a user, as shown in Figure 4.

As shown, the interactions of the application and user provide four possible combinations of what the user and the application know about the user's intentions: (a) explicit - user's intentions that are known to the user and application; (b) Inferred - information about intentions that the application infers but which are not explicitly part of the user's intentions; (c) implicit -intentions known to the user but not to the application; and (d) hidden - intentions that are not known to either the application or to the user. These four quadrants provide a valuable base point in which to separation of design issues in relation to context-aware applications.

\section{Contrasting Definitions, Categorizations, and Models}

Some definitions and categorizations of context are too specific, as they do not allow sufficient flexibility for different situations and applications (e.g., Coutaz \& Rey, 2002; Schilit \& Theimer, 1994). This view is also shared by Dey and Abowd (1999), who stipulated that "context is about the whole situation relevant to an application and its set of users." For all types of situations, it 
Figure 4. Interactions of Application and User (Zetie, 2002a).

\begin{tabular}{lcc}
\hline & Unknown to the User & Known to the User \\
\hline Known to the application & Inferred & Explicit \\
Unknown to the application & Hidden & Implicit \\
\hline
\end{tabular}

is impossible to stipulate what aspects will be pertinent. Using a mathematical model of context, for instance, may restrict the likelihood of being able to fully capture a user's complex interactions with other people and objects in his or her environment. This explanation also demonstrates why it is dangerous to use the application as the primary focus when defining context. To maximize usability, users and their heterogeneous interactions need to be placed at the center of a design process; otherwise, systems will become negligent of users' requirements, making them obtrusive and frustrating to use.

Some definitions are also too general and nonspecific (e.g., Dey \& Abowd, 1999; Funk \& Miller, 1997; Schmidt, 2001). They imply that context is anything that is relevant (or matters) to an entity or interaction of entities. Because it is not explicitly described how relevant dimensions of context can be identified and quantified, it would be very difficult to transfer or operationalize these definitions into a complex contextual scenario. It would also be uneconomical and tedious to identify from scratch which entities are germane to more than one situation. Midway points are required whereby generic categories (or reference points) of context can be applied to every situation. The content, importance level, interactions, and influences of each category can be measured for each situational purpose.

A key issue that transpires from the previous two points is: How are dimensions of context identified, quantified, and interrelated for each situational purpose? Zetie (2002b) stipulated that to ensure the application adopts itself to the intentions of the user, task analysis is critical for a suitable and sufficient investigation.

\section{Proposed Summary Model of Context in Computer Science}

The contrasting viewpoints, definitions, and categorizations of context within the area of computer science have been illustrated in our model, which is shown in Figure 5. Whether the primary focus is from the perspective of the user, the application, or from any entity of interest, Figure 5 illustrates the key components and characteristics of context that are present during user-computer interaction (i.e., use context).

As shown, surrounding people and objects may influence the user-computer interaction, making it an intrinsic factor in shaping the use context. The broken 
Figure 5. Our proposed model of context in computer science.

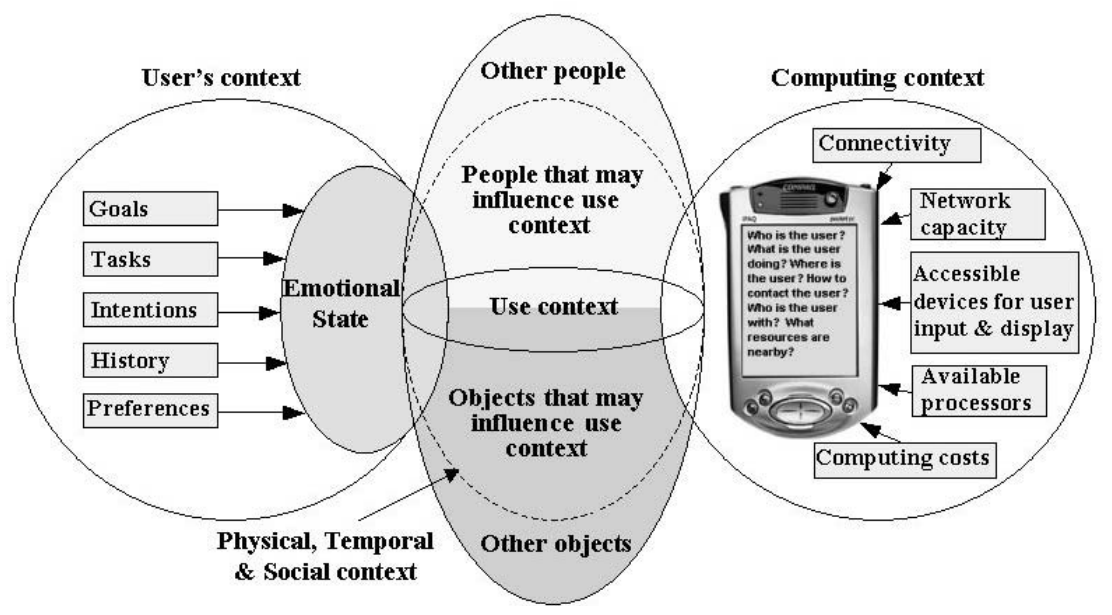

line demonstrates how, as a result of temporal changes, other objects and people may pass from being relevant or irrelevant to the interaction taking place.

\subsection{Psychology and Context}

Over the years, researchers in psychology have studied how changes in context can affect various cognitive processes, such as perception, language interpretation, reasoning, decision making (framing effects), problem solving (fixedness and set effects), learning (lack of transfer from one context to another), and memory (priming effects).

Many psychologists have attempted to define and categorize context. In relation to context-dependent memory, Smith (1988) defined context as "a concept that denotes a great variety of intrinsic or extrinsic characteristics of the presentation or test of an item." On the basis of previous research: Smith also contrasted two different conceptualizations of context:

1. Focal versus contextual: Focal information is directly in the focus of attention (e.g., likened to foveal vision), whereas contextual information is processed outside the focus of attention (e.g., likened to peripheral vision).

2. Meaningful versus incidental: Meaningful context refers to verbal/semantic material (e.g., accompanying words/text that may directly bias meaning selection processes). Incidental context is not meaningfully related in any implicit or explicit way (it just happens to be present). Kokinov and Grinberg (2001) demonstrated how perceiving some incidental objects from the environment may change the way in which we solve problems. 
In other viewpoints of context, Ziemke (1977) stated how context has been previously categorized into external (or objective) context and internal (or subjective/cognitive) context. External context would be the situation or environment the person is in, and the internal context would be the internal knowledge/mechanisms underlying the person's cognitive processes (e.g., mood, state-dependent effects). Bekerian and Conway (1988), however, added another dimension of context, namely, everyday context, which includes scripts or frames for action that prepare the person to expect subsequent events and to anticipate particular outcomes.

In relation to internal context, Ziemke (1997) distinguished between two major paradigms in contemporary cognitive science: (a) cognitivism and (b) enaction. They are described thus:

1. Cognitivism: In any specific situation, not all knowledge about the world is applicable, useful, or pertinent. The information solicited to make a cognitive process is therefore the subset of information that is available (e.g., acquired information regarding weather forecasts would be useful for planning holiday activities; however, it would not be useful for cooking a meal).

2. Enaction: Cognition is not considered an abstract human internal process; instead, it is an embodied process that is the outcome of the constant human-environment interaction (and their mutual relation during evolution/individual development). The cognitive processes required to effectively interact with a laptop, for instance, would have been influenced by previous computer interactions, during which skills, knowledge, and experience would have been gained.

Last, Davies and Thomson (1988) provided an interesting insight into the impact of context on memory. On the basis of investigations into environmental context (EC) dependent memory, two types of effects were identified. Long-term reinstatement effects were the memories experienced when returning to a former residence after a long absence (e.g., often triggered by important lifestyle changes, such as marriage, divorce, emigration, etc.). Short-term EC effects relate to the familiarity of people/objects in particular situations/environments. If encountered in another environment, it is common not to recognize this person/object in this new context.

\section{Usability Issues for Context-Aware Applications}

Combining the enaction paradigm (Ziemke, 1997) with the investigations into EC-dependent memory (Davies \& Thomson, 1988) reveals an important issue for context-aware computing. The embodied mutual evolution of the human-environment process must be tracked by the application to provide information and services that are suited to the user's memories of past experi- 
ences. For instance, a tourist called Bob is visiting Rome for his second time. Since his previous trip, certain features of the EC may be familiar (i.e., long-term reinstatement effect). To provided tailored functionality of contextual detail, the application must determine from its own memory (a) the length of time since Bob's last visit, (b) the information that was provided to him, and (c) his previous activities. A key question would be: How does the application account for variabilities in human memory (e.g., a decline in cognitive function due to age)? A possible solution would be to use a memory-triggering process involving snippets of information.

There may also be instances when it is useful to provide information about EC that has changed since a previous visit (e.g., buildings erected, shops and restaurants changed, etc.). If Bob were blind, for instance, and depended on building a comprehensive cognitive map ${ }^{2}$ or internal representation of the environment, the mobile device may need to inform him of EC modifications to ensure he does not become disorientated or confused in his surroundings as a result of information being conflicting to his memory (i.e., short-term reinstatement effect). This could be achieved by contrasting the geographic application database used during Bob's last visit with a new, updated version. Providing such feedback enhances the development, evolution, and mutual relation of humans with their environment.

The differentiation between incidental and meaningful context also has a major design implication for context-aware applications. If the application senses a nearby art gallery (unknown and incidental to Bob), while directing him to the train station (his meaningful task), how should this information be presented (if at all) and prioritized with respect to his current task and personal preferences? For the application to make those types of inferences, the following parameters may need to be accounted for: the time available before his train arrives, whether he has visited galleries before, and so on.

\section{Proposed Summary Model of Context in Psychology}

On the basis of the conceptualizations and theories of context within the psychologist's domain, we propose the following summary model of context, shown in Figure 6.

The model is composed of an inner and outer circle forming the focal and contextual layers. One line splits the circles perpendicularly into internal and external contexts, although the other line splits the circles horizontally into

${ }^{2} \mathrm{~A}$ cognitive map is an interpretive framework of the world which, it is argued, exists in the human mind and affects actions and decisions as well as knowledge structures (see http://www.hyperdictionary.com). 
Figure 6. Our proposed model of context in psychology.

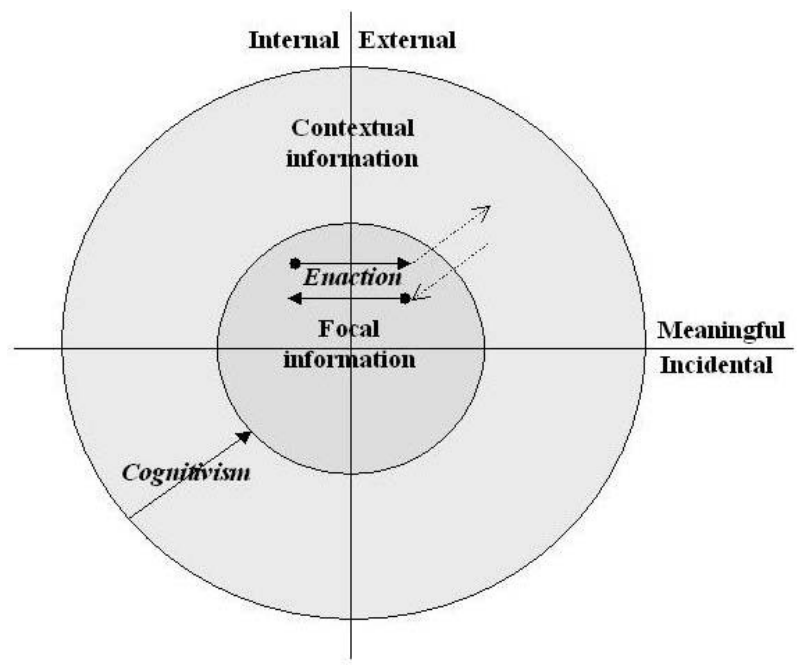

meaningful and incidental contexts. As illustrated in Figure 7, the following scenario, involving Bob en route to the Coliseum in Rome using his GPS-enabled Palmtop for navigational assistance, is used to illustrate the eight possible combinations.

Bob's scenario can be used to illustrate the enaction paradigm, shown in Figure 6. Within Bob's internal context, decisions that are focal and meaningful are made prior to, and during, his trip. These decisions are influenced, changed, and created by his interaction with focal and contextual information within his external context.

The cognitivism paradigm can also be illustrated using Bob's scenario. If Bob had extensive knowledge of Spanish and Italian culture and history, only his Italian knowledge (a subset of his total knowledge) would influence his cognitive processes or imminent decisions. If Bob, however, had been in Madrid, the opposite would have occurred.

\subsection{Context Within Other Research Areas}

The notion of context has also been investigated within many other areas of research, including artificial intelligence $(\mathrm{AI})$, neuroscience, pragmatics, and philosophy. Researchers in AI, for instance, have recently attempted to formalize context in order to model it in computer simulations. They are predominantly interested in how context is connected to reasoning, for instance, how people change their perspective, their line of reasoning, how they think of and compare 
Figure 7. A scenario depicting eight possible combinations in psychology.

\begin{tabular}{lc}
\hline Scenario & Possible combination \\
Bob wishes to navigate to the Coliseum in Rome. & Internal-Meaningful-Focal \\
He solicits trip information from the IPAQ display & External-Meaningful-Focal \\
using his stylus. & $\begin{array}{c}\text { External-Meaningful-Contextual } \\
\text { External-Incidental-Contextual }\end{array}$ \\
As Bob interacts with his IPAQ, he is aware of & \\
someone approaching him in his peripheral vision. & External-Incidental-Focal \\
This person asks Bob for the time, which he gives. & Internal-Meaningful-Contextual \\
Bob returns to his IPAQ, selects the shortest route, & \\
and is soon on his way. Whilst walking, Bob & \\
decides that he would be keen to visit any art & \\
galleries en route to the Coliseum. & \\
After a while, Bob starts to feel hungry but decides to & Internal-Incidental-Contextual \\
continue his journey to the Coliseum. & \\
Not long after, however, his hunger deepens and so & Internal-Incidental-Focal \\
he decides to eat at the nearest restaurant. & \\
After lunch, Bob is on his way again and eventually & \\
arrives at the Coliseum. & \\
\hline
\end{tabular}

states of the world in different situations or moments of time. See Theodorakis and Spyratos (2002) for a detailed discussion of issues of context in AI.

Neuroscientists, however, have explored the differences between implicit and explicit memory and learning, shedding light on the mechanisms that process information in the human brain. A number of differences have been discovered, as described by Tijus (2001), between explicitly and implicitly represented information and its processing and storage.

\section{CONTRASTING AND ANALYZING PROPOSED MODELS}

The proposed models of context for linguistics, computer science, and psychology (described in Sections 2.2-2.4) will now be critically analyzed and compared, in order to propose a multidisciplinary model in Section 4 . This assessment has been captured under two headings relating to contextual interactions and the notion of relevancy. The last section proposes a multidisciplinary definition of context based on Sections 2 and 3.

\subsection{Contextual Interactions}

Contextual interactions appear to comprise the quintessential cross-disciplinary component for understanding and using principles of context. From a high-level perspective, within linguistics it is the interaction between two people; within computer science it is the user-application interaction (combined 
with possible interactions with other people and objects), and within psychology it is the internal and external interactions.

Despite the fact that each discipline has contrasting viewpoints of context, their principles can be conceptualized across one another, which, although adding greater complexity, provides a deeper understanding in how people interact with the environment. A person speaking to a friend using his mobile phone, for instance, is both making an interaction with his mobile device at the same time as he is constructing dialogue that has been filtered through a cognitive, task, social, physical, and temporal context. So, in this example, the linguists would be interested in how a speaker changes his utterances during phone-based dialogue compared with human-human dialogue. The computer scientists would be interested in how this context-driven communication influences the design of the user interface as well as to the services and information provided to them, and the psychologists would be interested in the cognitive processes by which knowledge is acquired, stored, selected, and used to both interact with the mobile phone and converse with the friend. Each discipline has therefore implications for the other two disciplines.

To address an important comment made in the beginning of this article, each discipline is tackling different representations of context but at the same time can be considered as addressing similar, overlapping, and complementary themes of the same problem, perhaps the most important of these being: How do people decide what aspects of their cognitive, task, social, physical, and temporal context are relevant to them when undertaking, or planning for, future activities?

Last, contextual interactions should also be considered through the notion of embodiment, as described by Dourish (2001), whereby anything that provides a presence and participation to an activity or action (e.g., physical objects, conversations, actions, cognitive process, etc.) needs to be accounted for. This enables one to explore the social, cultural, organizational, and interactional context in which actions emerge.

\subsection{The Notion of Relevancy}

Issues of context and relevance are frequently cited in the science and philosophy literature. Although they are normally treated separately as unrelated topics, Ekbia and Maguitman (2001) argued that context and relevance are inextricably linked and should be analyzed together within a framework of logic. Dewey (1931) provided a pragmatic definition of context (in relation to relevancy) and differentiated between two components:

1. Background: Includes spatial and temporal dimensions and is ubiquitous in all thinking (e.g., "spatial" in that it covers the entire environment in which 
a thought emerges). Background is considered to be that part of the context that "does not come into explicit purview, does not come into question; it is taken for granted."

2. Selective interest: Context is considered to dictate the person's thought process. The notion of relevancy arises when the theory of selective interest occurs. There is evidence to suggest that humans take a selective interest in information (consciously/unconsciously) that is considered to be a cognitive benefit, either to enhance their knowledge or to use this resource to carry out specific activities (Matsui, 2001).

As depicted in each of the proposed models for linguistics, computer science, and psychology (see Figures 3, 5, and 6), the notion of relevancy is of critical importance for all disciplines when attempting to understand human behavior. So, within a communication act, illustrated in the linguistics model, background and selective interest would refer to the process by which humans take selective interest in both their own background cognitive purview (selecting relevant aspects of previous experiences and knowledge) and their background environmental situation (involving the person with whom they are conversing as well as their social and physical surroundings) in which to form communicative and noncommunicative goals.

This concept can be extended when considering the model of context for psychology. The selective interest process (i.e., determining what is relevant) may have been meaningfully or incidentally motivated, possibly resulting in different conversations and levels of significance/relevance. For instance, a student who visits a lecturer at the lecturer's office on the spur of the moment, compared with a student who prearranges a meeting with that lecturer, may have entirely different conversational and situational outcomes.

A user's interaction with a mobile device also illustrates these concepts. A person may take selective interest in surrounding people or objects (either meaningfully or incidentally) that may have been previously regarded as background context. This also nicely demonstrates one of the design challenges for context-aware computing: Systems must, in a sense (i.e., from a physical interaction perspective), be pushed into the background so that the user can focus and freely take selective interest in, and learn about, other people and objects in his or her environment.

\subsection{Proposed Multidisciplinary Definition of Context}

On the basis of Sections 2 and 3, we propose the following multidisciplinary definition of context. To make the definition useful, context is considered a process, rather than a product. 
Context: a process whereby a person consciously or unconsciously compares an external context with acquired personal experiences/knowledge (both of which may contain task, physical, social, and temporal dimensions) to form goals for undertaking concise actions, possibly with other people and/or objects.

\section{PROPOSED MULTIDISCIPLINARY MODEL OF CONTEXT}

Two versions of our multidisciplinary model of context are presented: (a) an outline version (see Figure 8) and (b) a detailed version (see Figure 9; Bradley \& Dunlop, 2003). The model captures the relationship between different interpretations of context by researchers within psychology, computer science, and linguistics. The key principles are described under three headings, concerning (a) the differentiation of the user and application's world, (b) the separation of meaningful and incidental dimensions of context, and (c) the user and application's processes.

\subsection{Differentiation of User and Application's World}

The horizontal center line shown in our outline model in Figure 8, separates the user's world from the application's world.

The oval-shaped circle in the center of Figure 8 represents what is focal to the:

1. User with respect to carrying out actions in an attempt to achieve goals (e.g., interact with Palmtop to find the time of a train home, interact with self-service ticket machine to acquire a train ticket). Goals and associated actions may be interrelated and form part of a higher level structure or goal (e.g., to get home). Actions can also occur independently or simultaneously.

2. Application with respect to transmitting contextual information and services to the user (e.g., alerting the user that a friend is in a nearby café).

In contrast, the circular layer that surrounds the user and application's world represents anything in the contextual world that influences the process in which focal user actions are undertaken and focal application services are executed. The contextual world can be broken down into several dimensions, four of which are common to both the user and application and are described as follows:

- Task context: The functional relationship of the user with other people and objects and the benefits (e.g., resources available) or constraints (e.g., time pressure) this relationship places on the user' achievement of his or her goal. 
Figure 8. Our outline model of context.

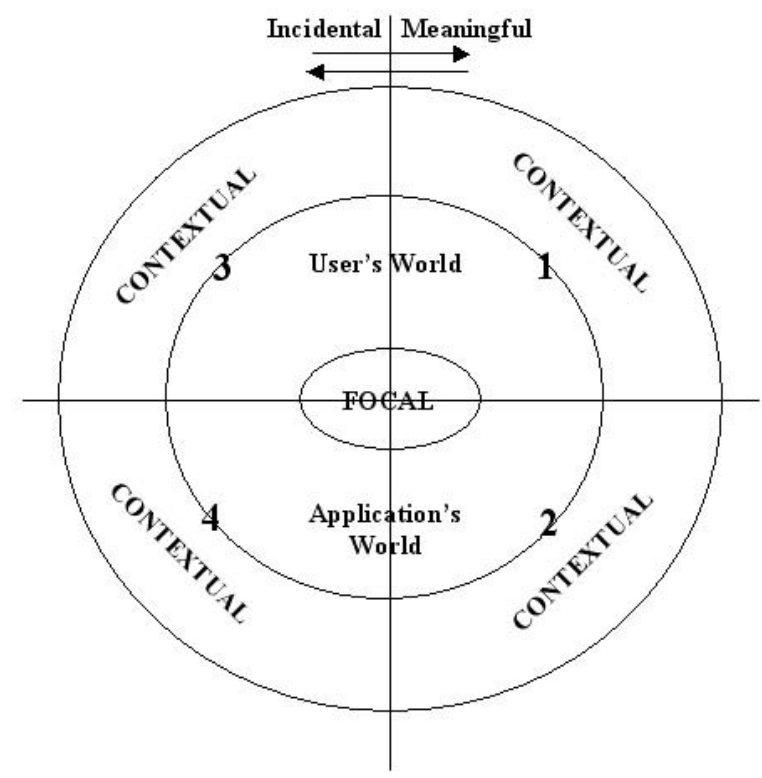

- Physical context: The environmental location, including its gradient and altitude, consisting of surrounding physical objects, such as buildings, cars, trees, and so on. This also includes the orientation, position, state, and purpose of those objects and the types of information they transmit through audio and visual means, odor, texture, temperature, and movement. Contrasting weather conditions (e.g., cloudy or sunny, cold or hot, etc.) and lighting conditions (e.g., daylight or darkness) may also influence how objects are perceived.

- Social context: The relationship with; dialogue from; and density, flow, noise, and behavior of surrounding people (e.g., sitting on a crowded train).

- Temporal context: The temporal context is embedded within everything (as illustrated in Figure 9) and is what gives a current situation meaning, based on past situations/occurrences; expected future events; and the higher level temporal context relating to the time of day, week, month, or season.

Another contextual dimension affecting the user's world is the application's context, which to the user would concern transmitted information regarding focal application services and his or her perception of the application's capabilities or limitations and of how it operates. Although part of the user's world, the cognitive context also affects focal actions, as described next. 
Figure 9. Our detailed model of context.

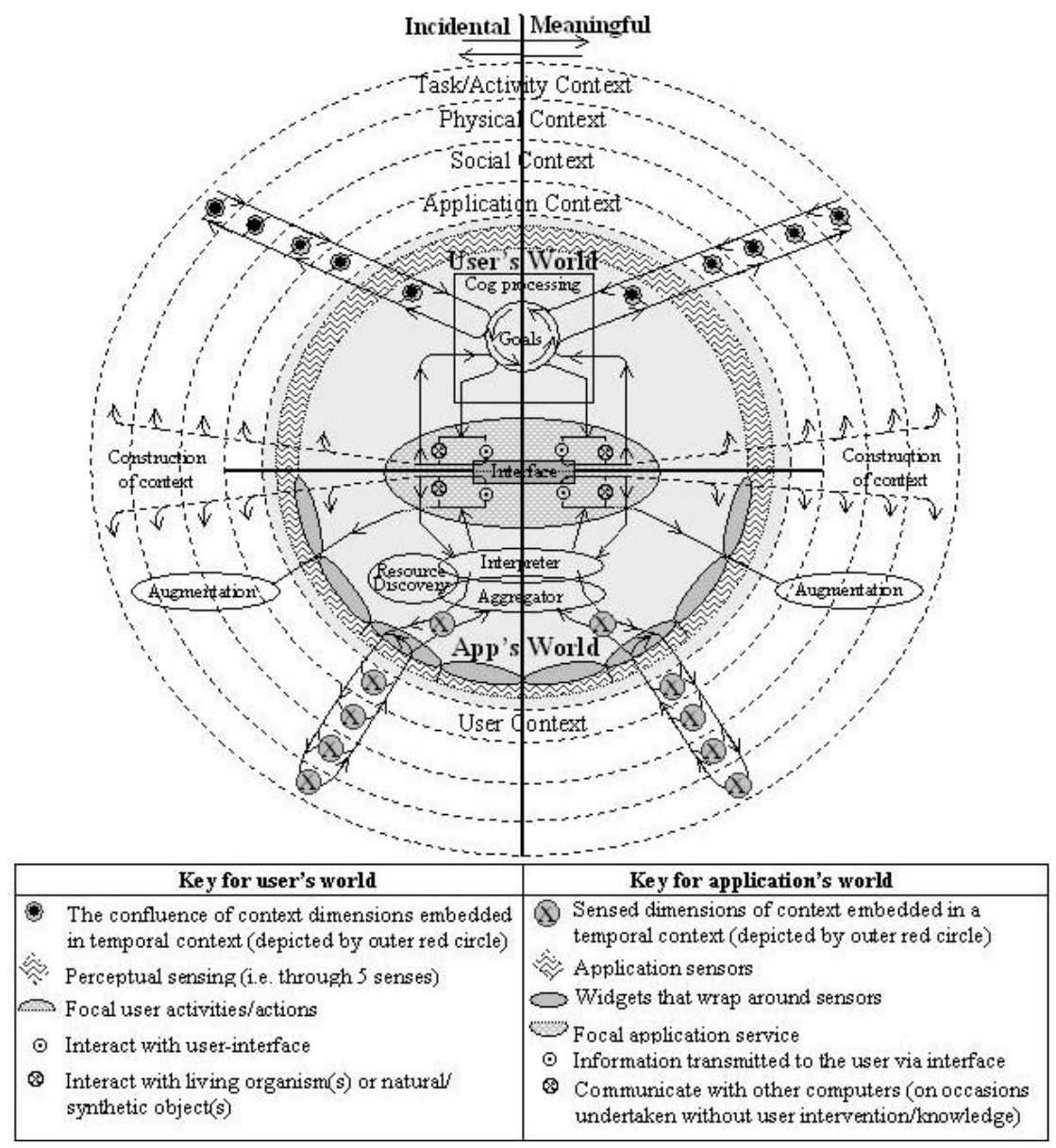

- Cognitive context: A user's cognitive processing abilities, short- and long-term memory abilities, dislikes and preferences, opinions and beliefs, cultural interpretations, perceptual sensing abilities, cognitive mapping strategies for encoding spatial information, and so on.

Within the application's world, another contextual dimension that could be sensed is the user's context. This may include information regarding (a) the user's personal diary, including planned activities, notes, and reminders, as well as user-defined application settings and preferences; (b) physiological sensing, such as heart rate, to measure levels of anxiety; and (c) monitored behavioral patterns of the user. Similarly, although part of the application's 
world, the application context also affects how focal services are executed, as described next.

- Application's context: The capabilities and limitations of both the application (e.g., battery usage life, processor speed, memory capacity, sensors, input and output technologies, etc.) and the sources from which data are derived (e.g., the processing speed of a Web-based server).

\subsection{Separation Between Meaningful and Incidental Dimensions}

The next separation to make is between incidental and meaningful context, as depicted by the center vertical line in Figure 8. Generally speaking, meaningful context is implicitly related to the user's primary high-level goal, whereas incidental context is concerned with incidental occurrences in the contextual world that are normally unrelated to the user's primary high-level goal (e.g., bumping into a friend, being caught in a sudden downpour, etc.). To illustrate in more detail, we describe each of the four quadrants separately.

\section{Quadrant 1}

The user is undertaking meaningful focal actions (e.g., interacting with his or her Palmtop) and is using, or being positively or negatively influenced by, meaningful contextual dimensions in order to realize his or her primary high-level goal (e.g., influenced by the surrounding people while he or she is on a busy train). This quadrant is similar to the techniques of task analysis, which are aimed at eliciting the structured set of meaningful actions or activities people carry out to accomplish an explicit goal or task (see Preece et al., 1994).

\section{Quadrant 2}

The application is aware of (or thinks it is aware of) the user's primary high-level goal (either inferred by the application or explicitly given by the user) and uses sensed data acquired from the contextual world to execute a meaningfully focal service (e.g., informing the user that the train home is delayed).

\section{Quadrant 3}

Incidental occurrences in the contextual world are normally unrelated to the user's primary high-level goal. These events may either remain incidentally 
contextual if they have no impact on the user's meaningful activities (e.g., other people walking past), become incidentally focal if the user needs to temporarily deviate away from his meaningful activities (e.g., has to cross the street to navigate past road work), become meaningfully contextual (e.g., decides to walk another route on subsequent days), or become meaningfully focal (e.g., if the person badly injures himself by falling down a hole in the road).

\section{Quadrant 4}

The application uses sensed contextual data, similar to Quadrant 2, to either support incidental focal events (as described in Quadrant 3) or infer future user intentions of which the user may be currently unaware (e.g., informing the user of a friend in a nearby café). The application may also acquire sensed data and discover resources and tools in the environment that, although perhaps unrelated (and therefore incidental) to the present high-level goal, are considered of potential benefit to the user in future meaningful activities (e.g., a tool to provide a weather report whenever a new city is visited).

\subsection{Application and User Processes}

The space between the center focal circle and the inside perimeter of the contextual layer in Figure 8 concerns the user and application processes that link the contextual world to the focal world and differentiate the incidental and meaningful worlds. This is illustrated in our detailed multidisciplinary model of context shown in Figure 9.

In general, the depiction of the user's world in Figure 9 illustrates how cognitive goals of a user are continually shaped by people's perception of the meaningful and incidental contextual worlds, consisting of task, physical, social, application, and cognitive dimensions, all of which are embedded in a temporal context. These goals are used to carry out concise focal actions, which may involve interactions with the application or surrounding people and objects. Focal interactions shape the context within which subsequent interactions take place and also contribute to the user's construction of future goals.

The depiction of the application's world illustrates how the application senses, stores, and interprets contextual data. Focal services, the purpose of which may be to transmit information to the user, leave information at a specific location in space (i.e., contextual augmentation), or communicate to other computers, possibly without the user's knowledge or intervention, are then executed. Similarly, these focal services construct the application's context and the context within which the application senses.

To explain in more detail the key concepts in Figure 9, we explain the user and application processes separately. 


\section{User Processes}

Within the meaningful world, shown in the top right quadrant of Figure 9 (Quadrant 1 of Figure 8), the user utilizes, or is influenced by, dimensions of the contextual world. We now describe this process in more detail using a scenario illustrated in Figure 10.

Shown in the "Goals" circle on the far left, the user has a high-level goal of catching the last train home. This goal would be broken down into a series of low-level goals - the current one being to purchase a train ticket. When attempting to realize this goal, the user is selecting meaningful and relevant aspects of the external contextual environment, such as the option of a manned counter and ticket machine. In contrast to selection of context, the user is also being influenced, either positively or negatively, by the external contextual environment (e.g., the manned counter closing in $10 \mathrm{~min}$ - so in this case the arrows in Figure 10 would start from the right).

In either situation, meaningful aspects of the contextual environment, all of which are embedded in a current, past, and present temporal context, are perceived by the user through the five human senses, namely, sight, hearing, smell, touch, and taste. The information is then interpreted through the user's cognitive context (e.g., intolerant of queues and highly stressed) leaving a perceived meaningful representation of the external world that is compared with relevant past experiences or acquired knowledge. The user then makes a decision regarding the most appropriate goal with which to undertake a concise, meaningful focal action. After the user has executed a focal action, this both forms an experience with which future perceived cognitive representations of the external environment can be compared and constructs the context within which future contextual interactions take place as shown in Figure 9.

As shown in Figure 9, the user also contains an incidental world, where objects, people, and/or thoughts occur incidentally and are normally unrelated to the high-level meaningful goal (e.g., passing a newsstand in the train station). Similar to the meaningful world, these incidental occurrences have an embedded temporal context and are perceived by the user through his or her cognitive context, leaving an internal representation of the incidental world. Unlike the meaningful world, cognitive processing here involves a conscious or unconscious assessment of whether this information is a cognitive gain or necessity (e.g., to negotiate a particular hazard). This evaluation will be influenced by the user's previous experiences or acquired knowledge (similar to the meaningful world), resulting in the user's decision to either discard this incidental occurrence altogether, form a goal to undertake an incidental focal action, or make the incidental occurrence a meaningful contextual influence (e.g., the newsstand is used to acquire change for the self-service ticket machine). 


\section{Figure 10. User processes that link the contextual world to the focal world.}

\section{Scenario: To purchase a ticket at the train station}

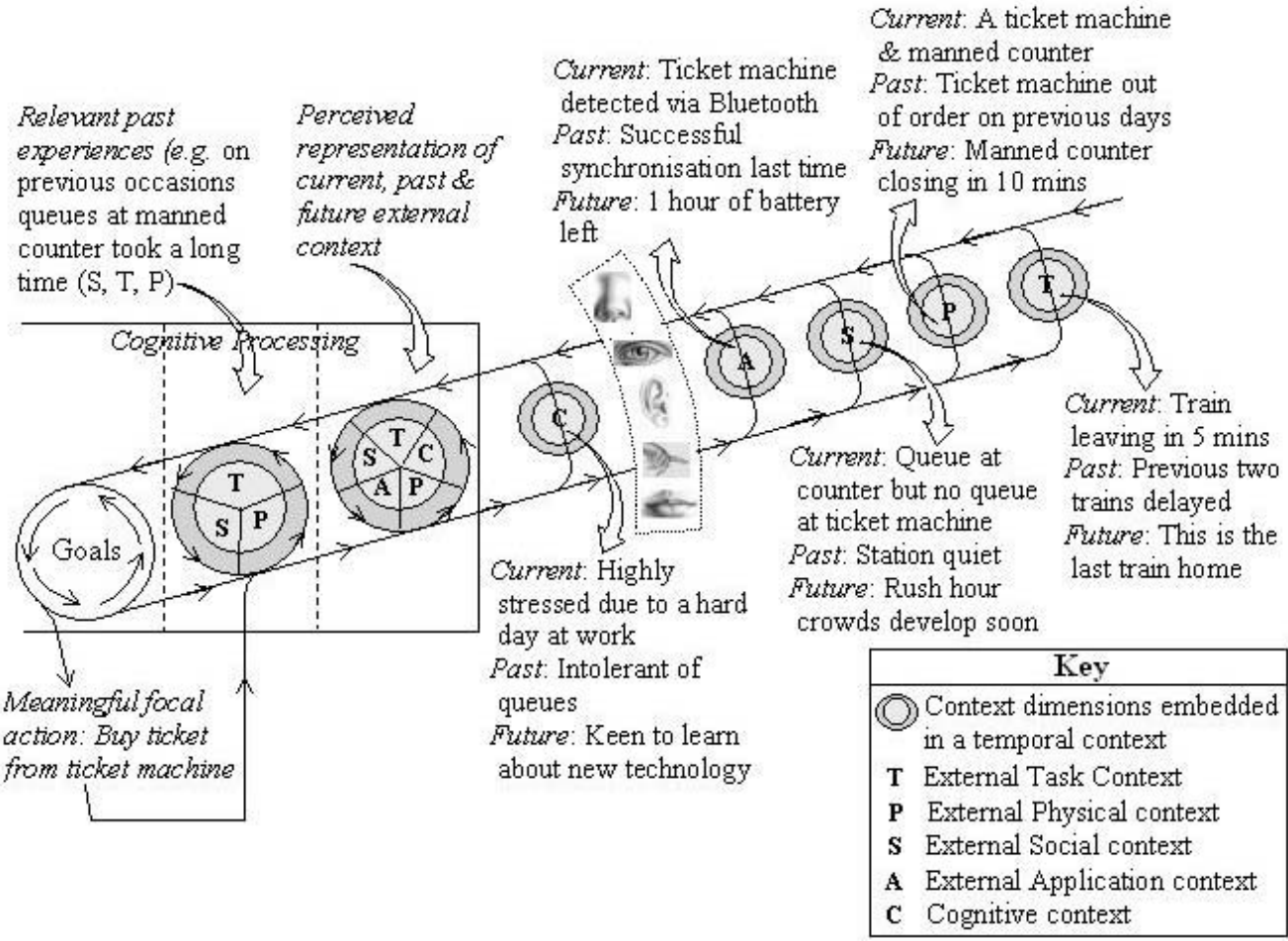


A feature not captured in the model is the way in which interactions of objects and people influence the context within which users interact and make decisions. For instance, if people over a period of time leave electronic reviews of the same restaurant, how do these combined reviews influence users' decisions on whether to eat there? This type of issue is captured in our design framework.

\section{Application Processes}

Dey, Salber, and Abowd's (2001) component-based conceptual framework for building context-aware applications is partially used to represent the application's world shown in Figure 9. This framework was chosen because it represents a significant milestone in ubiquitous computing and has been used as an anchor article for a special issue on context-aware computing (Moran \& Dourish, 2001).

The context abstractions used were widgets, aggregators, and interpreters. The processes within the aggregator and interpreter, and the flow of context data, have also been represented slightly different to illustrate the key principles of Figure 9. In essence, widgets represent sensor abstractions that conceal details of how sensing and interpretation of the environment occurs. Widgets essentially wrap around underlying sensors and provide an interface to automatically deliver information to interested components or services of the system. Aggregators store multiple pieces of low-level information (e.g., a person or location) that is logically related and stored in a common repository for relevant application entities. Interpreters are responsible for abstracting low-level data to higher level information (e.g., using the user's location, time of day, and travel velocity to infer that he or she is on the train home from work). The two remaining context abstractions of Dey et al.'s (2001) framework, namely, services and discoverers, are not shown but would be included within the focal application service section of Figure 9. Discovers are responsible for maintaining a registry of what capabilities exist in the framework, and services are the same as context widgets, except the output is abstracted and the actuators or change of environmental state information is controlled.

We now describe in more detail the processes of collecting, transforming, and delivering contextual information, as illustrated in Figure 11.

Figure 11 illustrates how the context-aware system senses meaningful aspects of the contextual world, the dimensions of which are embedded within a past, current, and future context. The availability and accuracy of sensed data, however, will be dependent on the application's context (i.e., the capabilities and limitations of the application and the sources from which the information was derived).

Our representation illustrates how data are then aggregated and logically stored under five common repositories relating to the user, task, physical, so- 


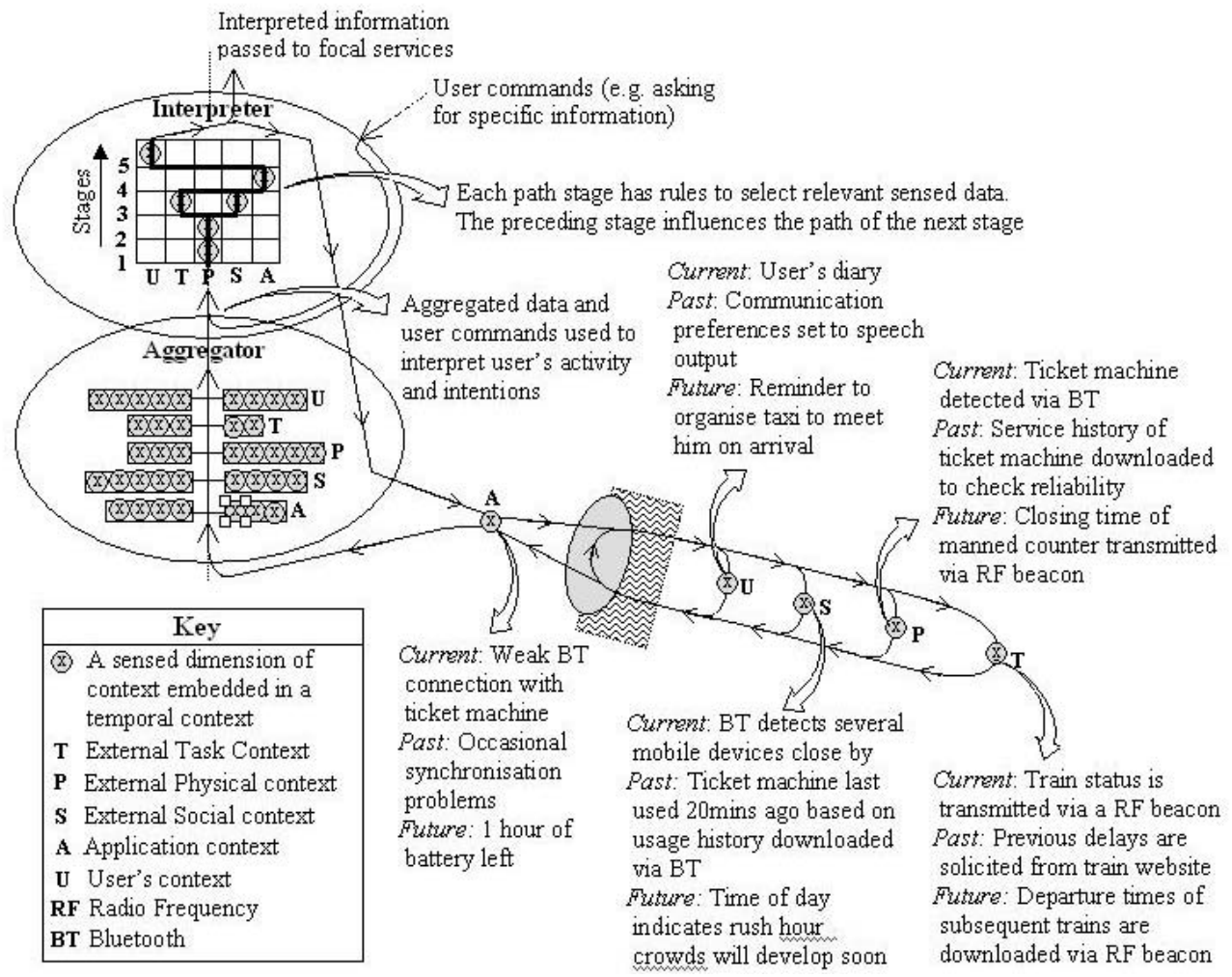


cial, and application context. Aggregated sensed data contain past, current, and future dimensions and can be used to interpret meaningful and incidental activities of the user. The application's incidental world, shown in Figure 9, also contains a resource discovery. Here, tools in the environment are discovered and (if inferred to be relevant to the user) are downloaded to enhance its functionality and robustness. These tools may be used to either support incidental or meaningful application processes.

Within the interpreter, shown in Figure 11, our representation illustrates the conceptual process in which aggregated data could be abstracted (either driven by commands of the user or application). This has been depicted using a matrix (the characteristics of which are identical for both incidental and meaningful processes) consisting of the dimensions of aggregated sensed data on the horizontal axis and the different levels/stages of abstraction on the vertical axis. The path through the matrix is governed by rules (possibly derived by previous paths or from application design) that are used to select relevant sensed aggregated data for each subsequent stage. The following superficial example, as depicted in Figure 11, is described in the following sections.

Stage 1. Physical aggregated data-the user located in the train station. Rule: From previous matrix paths, the user typically decides to buy a train ticket when entering a bus or train station. Find out what objects are nearby.

Stage 2. Physical aggregated data-a ticket machine and manned counter. Rule: From previous matrix paths involving Stages 1 and 2, the user typically uses the counter to buy his ticket, although if there is a queue and time is pressing, he uses the ticket machine. Find out the status of train and queues at counter and ticket machine.

Stage 3. Task and social aggregated data-train leaves in $5 \mathrm{~min}$, there is a queue at counter and no queue at the ticket machine. Application infers that user will wish to use the ticket machine. Rule: From previous matrix paths involving Stages 1, 2, and 3, the application has had Bluetooth connection problems with the ticket machine. Find out strength of Bluetooth signal.

Stage 4. Application aggregated data-Bluetooth signal is strong. Rule: From previous matrix paths involving Stages 1, 2, 3, and 4, the user pays for his ticket either by using hard cash or by direct debit using Bluetooth. Find out what payment preferences the user has set.

Stage 5. User-aggregated data—user has set his payment preference as direct debit. This path is sent to the focal application services section, which results in an executed action. 
Once aggregated data have been abstracted or interpreted, the path through the matrix, along with the selected sensed data, is passed to the focal application service section, as shown in Figure 9. Focal services can take one of four forms: (a) task-specific information, either inferred by the application or requested by the user, is presented to the user via the user interface (e.g., the application meaningfully infers that the user is catching a train home and informs him or her of a delay); (b) information is not presented to the user, although the application executes a focal service as directed by the user (e.g., the user could augment, as shown in Figure 9, the environment at a precise location, informing friends of a good bookstore); (c) the application meaningfully or incidentally infers a particular course of action without the user's intervention (e.g., automatically calling the user's doctor if he or she were to become ill); or (d) an application service is executed to facilitate other application services (e.g., the application downloads a software tool for obtaining football scores, in order to more accurately infer a user's likely course of action- to the pub to celebrate!).

\section{APPLYING THE MULTIDISCIPLINARY MODEL TO DIFFERENT APPLICATIONS OF CONTEXT-AWARE COMPUTING}

Dey and Abowd (1999) defined the essence of context-awareness or context-aware computing as "the use of context to provide task-relevant information and/or services to users wherever they may be." In other words, context-aware systems sense information within the user's context (e.g., location, time of day, nearby people and objects, personal preferences, etc.) to support, enhance, and predict a user's mobile activities, thereby minimizing the need for traditional human-computer interaction.

The purpose of this section is to illustrate how the concepts and principles of the proposed multidisciplinary model of context (described in Section 4) might apply to different applications of context-aware computing. Those included are mobile tourist guides and devices to support mobile user communities. An additional section at the end provides a superficial illustration of how an application developer might use our multidisciplinary model in practice.

\subsection{Mobile Tourist Guides}

Some researchers have been concerned with supporting the mobile information needs of tourists. GUIDE, for instance, is a mobile intelligent electronic tourist guide being developed at Lancaster University (Cheverst, Davies, Mitchell, Friday, \& Efstratiou, 2000). Personal computing technologies, wireless communications, context awareness, and adaptive hypermedia 
are integrated to support the information and navigation requirements of visitors to a city. Presented information is tailored to both personal (e.g., visitor's interests) and environmental (e.g, time of day) contexts.

In another system, CATIS (Context-Aware Tourist Information System) adapts its tourist information services using contextual elements such as location, time of day, speed, direction of travel, personal preferences, and device type (Pashtan, Blattler, Heusser \& Scheuermann, 2003). Brown (2000) also demonstrated how weather is an important factor in filtering relevant information, especially for ranking tourist attractions in accordance with current weather conditions.

Using a scenario involving a tourist named Alice whose high-level goal is to experience and learn about the art and culture of Glasgow, the following observations can be made with respect to our multidisciplinary model of context in Figure 9 (along with Figures 10 and 11).

User's meaningful world. Alice's lower level meaningful goal is to decide which attraction to visit. Her perceived cognitive representation of the environment includes a mixture of contextual information:

- Current physical context - A bus stop is $30 \mathrm{~m}$ away, positioned up a gradual incline. It is a cold day, and rain looks ominous.

- Current task and application context-Her Palmtop ranks the Hunterian and Lighthouse Art Galleries as the top two nearby attractions. The Lighthouse Art Gallery would take $35 \mathrm{~min}$ to walk to (with no option of public transportation), whereas the Hunterian Art Gallery could be reached in $15 \mathrm{~min}$ by bus, one of which is expected in $10 \mathrm{~min}$.

- Current social context-There is no queue at the bus stop.

- Past and current cognitive context-Alice dislikes walking, although she would slightly prefer Lighthouse Art Gallery.

Alice's representation is compared with her conversation with Bob (a relevant personal experience, as illustrated in Figure 10) who strongly recommended a visit to the Hunterian Art Gallery. Alice makes a decision and forms a goal to carry out concise meaningful focal actions to catch the bus to the Hunterian Art Gallery.

Application's meaningful world. The following aggregated sensed data are used by the application to interpret and support Alice's high-level goal:

- Current physical context-GPS location indicates that Alice is standing close to a bus stop in Glasgow. Temperature sensors indicate the current 
temperature is just above $0^{\circ} \mathrm{C}$. Rule: Check Alice's diary whenever she visits a new city.

- Current and past user context-Alice's diary indicates that she is on holiday. On previous holidays, Alice has visited art galleries and likes to be reminded of weather reports. Rule: Find out weather reports and what galleries are nearby.

- Current physical context-A Web-based geographical database indicates that the Hunterian and Lighthouse Art Galleries are nearby. A Web-based weather report reveals that intermittent heavy downpours are expected. Rule: Discover timetables of public transport.

- Future task context-Web-based public transport timetables indicate that the Hunterian Art Gallery can be reached in 15 min by bus, one of which is expected at the bus stop in $10 \mathrm{~min}$. No mode of transport to the Lighthouse Art Gallery was found, although a distance calculation and Alice's average walking speed indicate it would take around 35 min to reach by foot. Rule: Check Alice's preferred mode of inner city travel and check whether these preferences change in different weather conditions.

- Past user context-When it is a cold day, Alice prefers to travel by bus.

On the basis of this interpreted information, the application executes a focal meaningful service to rank a set of tourist attractions (along with methods of transport), having the Hunterian Art Gallery at top since Alice's preferred mode of inner city travel is by bus when it is cold day.

Incidental world of the user and application. There is a sudden downpour while Alice waits at the bus stop, which results in Alice taking cover at a shop entrance $15 \mathrm{~m}$ away while she waits for the bus (an incidental focal action). The application senses a change in Alice's location and infers from Web-based weather reports that Alice may have been caught in a downpour (or is at least likely to later on). Because the application discovers an umbrella shop that is en route to the next bus stop (approximately half a meter away), it calculates that Alice would have enough time to walk there, so it executes a focal service informing Alice of this information.

Usability issues. The application must prioritize which factors are likely to influence a user's decisions (transport convenience vs. attraction preference). The application must gauge or infer the likelihood of incidental events causing a change in a user's meaningful goal (e.g., if the incidental downpour had caused Alice to change her mind about visiting an art gallery). 


\subsection{Mobile User Communities}

Within the field of context-aware computing, some researchers investigate how communities of mobile users could be facilitated (e.g., Brown, 1996; Burrell \& Gay, 2001; Salber, Siewiorek \& Smailagic, 2001). The Stick-e document system, for instance, allows a user to place messages or Stick-e notes at various positions in space (Brown, 1996). These are detected, retrieved, and viewed by others, with location-sensing technology on a Palmtop, whenever this location is visited. The physical location of a stored message, however, can be extended to include other types of desirable context features, such as attaching notes about people or objects detected within a building (using active badges), triggering notes when specific states have been reached (e.g., temperature thresholds), and allowing users to create their own context-triggering conditions. In other work, Burrell and Gay (2001) described an application called Graffiti that is designed to allow a community of users to collectively specify what is relevant and useful about a location, situation, and identity. Posting electronic notes consisting of contextual knowledge (beyond what the system was able of detecting) would allow other users to receive information relating to that context.

This area of research would have obvious benefits for communities of visually impaired people, who could share experiences about different situations and environments (e.g., alerting others of hazards). Although this notion has not been exploited, there has been some research into supporting the navigational requirements of visually impaired people. The Drishti wireless navigation system (Helal, Moore, \& Ramachandran, 2001), for instance, integrates several technologies, including wearable computers, voice recognition and synthesis, wireless networks, GIS, and GPS, to provide contextual information and optimized routes based on user preferences, temporal constraints (e.g., traffic congestion), and dynamic obstacles (e.g., ongoing ground work).

Using a scenario involving a blind person named Bob en route to catch a train to Stirling for his friend's birthday, the following observations can be made in relation to user communities when applying our multidisciplinary model of context (illustrated in Figure 9 along with Figures 10 and 11).

User's meaningful world. Bob's lower level meaningful goal is to purchase a talking book for his friend's birthday before catching the train to Stirling. Bob's perceived cognitive representation of the external context includes a mixture of contextual information:

- Current and future physical context-Just around the corner from the train station, the bookstore that Bob is in has a special offer on talking books lasting for 3 days. 
- Future task context-Bob's train departs in 30 min.

- Current application context-While in the bookstore, Bob's Palmtop transmits an automated verbal message asking him if he would like to leave a message for other blind people about this special offer.

- Future social context-Bob feels this information might be of benefit to other visually impaired people.

- Current and future cognitive context-Bob is in a good mood and is not feeling time pressured. He considers the bookstore to have a good selection of talking books.

Bob's perceived representation of the external world is compared with his previous personal positive experiences of listening to talking books. He subsequently makes a decision and forms a goal to carry out concise, meaningful, focal actions to both buy a book for his friend's birthday and accept the application's inferred action to leave a message for other blind travelers.

Application's meaningful world. The following aggregated sensed data are used by the application to interpret and support Bob's meaningful goal:

- Current physical context-The GPS location indicates that Bob is in a bookstore in Edinburgh. Rule: Check Bob's diary to find out what his plans are.

- Future and current user context—Bob's diary indicates that he is due to attend his friend's birthday in Stirling. Bob has also created a note reminding him to buy a present. Rule: Bob frequently likes to take advantage of special offers, so check to see whether any offers are available.

- Current task context-A Web-based server indicates that there is a special offer on talking books for 3 more days. Rule: Check the profiles of Bob's friend and other members of the Web-based user community.

- Current social context-Bob's friend's profile indicates that she likes talking books, particularly adventure stories. Other members also indicate a strong appreciation of talking books.

On the basis of this interpreted information, two focal meaningful application services are executed: the first provides an inferred ranked list of talking books on offer for his friends present (having those relating to adventure stories at the top), and the second service provides an inferred inquiry asking Bob if he wishes to leave a message about this offer for other blind travelers.

Incidental world of the user and application. While walking to the train station, after having purchased his friend's present, Bob stumbles on a pothole that he was unable to detect in the pavement using his white cane. 
Bob undertakes an incidental focal activity to leave a verbal message warning other blind travelers of this hazard. The application asks Bob to specify a priority level to this message, to ascertain how much notice needs to be given (e.g., within a 5- or 10-m boundary?). The application infers that Bob may wish to send an e-mail to a local road maintenance authority at a later stage, so it stores the GPS location and executes a focal incidental service to remind Bob the next time he is checking his e-mail.

Usability issues. The application must enforce users to account for messages left for others (e.g., importance, usefulness, priority, and applicability of messages). For instance, messages regarding environmental hazards may need to be pushed to other visually impaired people (meaningful augmentation, as depicted in Figure 9), whereas messages regarding special offers may need to be presented less obtrusively, possibly deferring control of when information is viewed to the user (incidental augmentation, also illustrated in Figure 9).

\subsection{Applying the Model in Practice}

The purpose of this section is to provide a superficial illustration of how an application developer might use our multidisciplinary model in practice. Although its true value is dependent on its application at an early stage of development, the model can also be used to reinterpret existing applications or application areas to identify more robust and user-centered levels of support. In essence, the developer would need to ask questions regarding each quadrant of Figures 9 and 10, some of which have been listed in Figure 12.

\section{CONCLUSION}

When applying our model to different areas of context-aware computing, the true value of our multidisciplinary endeavor becomes apparent. From a high-level or holistic perspective, it allows application developers to develop richer scenarios and descriptions of how the mobile system may be used within various dynamic mobile settings. The model provides an augmentation to traditional task analysis, as the incidental interactions and occurrences in the mobile world, and not just the more predictable meaningful actions involved in accomplishing an explicit goal, can be investigated. As a result, more refined levels of user support can be mapped out, an exercise that will help application developers to design both meaningful and incidental services.

From a low-level perspective, the model can be used to investigate very specific issues of human behavior and application development, both of which are represented dynamically (i.e., context is a process). Within the model of the user's world, this includes both the contextual factors that influ- 
Figure 12. Issues and Questions to Consider When Applying our Model in Practice

Quadrant Questions about the user and application

1 (User's meaningful world) - After identifying the user's high-level meaningful goal, task, or activity, what types of scenarios would the user encounter?

- Within each scenario, what meaningful aspects of the contextual world is the user being influenced by when making decisions about focal meaningful activities?

- After focal activity, how does this construct the contextual world and user's cognitive context? How

2 (Application's meaningful world)

3 (User's incidental world)
- What types of focal meaningful services could the application execute to support a user's meaningful activities?

- What information within the contextual world would the application need to acquire, and how would this be sensed, stored, managed, interpreted, and transmitted to the user?

- After meaningful services have been executed, how does this construct the contextual world and application's context? What impact would this have on the user, on the environment, and on other people?

- What type of incidental events or scenarios might the user experience when undertaking his/her meaningful activity?

- What aspects of the contextual world is the user being influenced by when making decisions about incidental events? What type of focal activities might the user undertake?

- After focal activity, how does this construct the contextual world and user's cognitive context? How does this influence the user's future focal activities?

- What types of incidental focal services could the application execute to support a user's experience of incidental events? What additional incidental services could the application infer; information of which the user may be unaware of?

- What information within the contextual world would the application need to acquire, and how would this be sensed, stored, managed, interpreted, and transmitted to the user? How would incidental services be prioritised with respect to meaningful user activities and meaningful application services?

- After incidental services have been executed, how does this construct the contextual world and application's context? What impact would this have on the user, on the environment, and on other people? 
ence human decisions, spatial behavior, and focal interactions, and the subsequent construction of context within which future interactions take place. The model also helps address the issues of human variability in perception and cognition and helps tackle the unpredictable nature of users and the environments in which they interact. Within the model of the application's world, the value lies in the processes of identifying useful contextual information about the user; inferring human activity; delivering useful, relevant, and timely services; and monitoring the evolution of users and environments.

Another benefit of our model is on its focus of integration of the user and application's world. Issues can be considered together or in parallel, an activity that does not occur often enough in current application development and that can lead to more usable and unobtrusive systems. This helps developers to identify gaps and overlaps in knowledge, all of which can be used to draw out clearer and more seamless levels of support.

The main lesson that has been learned from the construction of our model is centered on the difficulty in representing context as a single model to cover the exhaustive viewpoints and interpretations that exist across and within disciplines. Although context is a complex subject that includes many wide-ranging issues, it is an extremely important area of research to mobile computing. Future mobile systems will be expected to operate in dynamic and contextually rich environments, and we feel that our proposed multidisciplinary model is sufficiently detailed and versatile enough to at least identify and investigate these issues further.

\section{DISCUSSION}

The primary aim of this article was to review and merge theories of context within the areas of linguistics, computer science, and psychology. The purpose was to propose a multidisciplinary model of context that could support the design of mobile, context-aware computing. When each discipline is analyzed individually, the usability implications are compelling. The differentiation between meaningful and incidental context in psychology, for instance, demonstrates how a user's decisions can diversify when other objects and people are encountered incidentally.

We found that, when disciplines are assessed collectively, each possesses similar, overlapping, and complementary characteristics that can be conceptualized across each other (despite tackling different representations of context). Further exploration is required in two particular areas:

1. An investigation of which aspects of the task, social, physical, temporal, and cognitive context are relevant to users when undertaking, or planning for, activities in various types of mobile scenarios. Procedures and techniques for 
tracking relevant contextual interactions need to be developed so that usability is placed at the center of context-aware design and development. These techniques would need to establish links between a person's unique cognitive and perceptual processes (e.g., people with visual impairments) and the presence, state, and purpose of static and dynamic objects in the environment.

2. An investigation of the temporal context with respect to its relationship with each dimension of context and how this relationship influences the process with which people form goals and carry out focal actions. For instance, a user's relationships with other people and objects change and evolve over time. For context-aware systems to adapt to such changes, a greater emphasis must be placed on multidisciplinary investigations to provide a deeper understanding of human behavior in mobile settings.

Summary models representing the opinions of researchers within each discipline were used to propose an outline and detailed multidisciplinary model of context. These are composed of four quadrants demonstrating the differentiation of focal and contextual aspects of the user and application's world, the separation of meaningful and incidental dimensions, and the user's cognitive processes and the processes of the application. The proposed model provides a foundation with which complex mobile scenarios can be conceptualized and modeled. We illustrated its applicability, versatility, and effectiveness to different applications of context-aware computing using examples including user communities and mobile tourist guides. Usability implications for both applications revealed important human and social issues, which are currently being captured in the development of our multidisciplinary design framework for context-aware computing. Issues would include the following:

1. The presentation of inferred incidental focal application services needs to be prioritized with respect to the user's focal activity and high-level goal (e.g., a blind person may not want to be pushed with information about a friend in a nearby café as he or she crosses a busy pedestrian crossing). Factors such as cognitive workload would need to be considered.

2. Users who wish to disseminate messages at specific locations for others to retrieve may need to prioritize their messages with respect to how they are transmitted to others. For instance, recommending a bookstore to a community of visually impaired travelers would be given low priority, whereas warning of a dangerous hazard would be given high priority (and possibly pushed to them).

To conclude, multidisciplinary models of context have been proposed to facilitate the design of more usable context-aware devices. This has been a valuable exercise, the findings of which provide a foundation in which complex mobile scenarios can be conceptualized and modeled. More 
multidisciplinary investigations are, however, required particularly into how the dimensions of context are composed and interrelated. This will reveal pertinent human and social issues, all of which will need to be resolved before such systems are to seamlessly integrate into people's mobile lives. Future work involves using our multidisciplinary models of context to develop a user-centered design framework. This will be used to design a large-scale user study investigating the usability issues of a context-aware mobile computing navigation aid for visually impaired people.

\section{Notes}

Background. This article is based on the Ph.D. thesis of the first author.

Authors' Present Addresses. Nicholas Bradley, Department of Computer \& Information Sciences, University of Strathclyde, Glasgow, Scotland, G1 1XH; Email: Nick.Bradley@cis.strath.ac.uk. Mark Dunlop, Department of Computer \& Information Sciences, University of Strathclyde, Glasgow, Scotland, G1 1XH; Email: Mark.Dunlop@cis.strath.ac.uk

HCI Editorial Record. First manuscript received March 4, 2003. Revisions received April 26, 2004, and October 28, 2004. Accepted by Gregory Abowd. Final manuscript received March 8, 2005. -Editor

\section{REFERENCES}

Bekerian, D. A., \& Conway, M. A. (1988). Everyday contexts. In G. M. Davies \& D. M. Thomson (Eds.), Memory in context; context in memory (pp. 305-318). Chichester, England: Wiley.

Bellotti, V., \& Edwards, K. (2001). Intelligibility and accountability: Human considerations in context-aware systems. Human-Computer Interaction, 16, 193-212.

Benerecetti, M., Bouquet, P., \& Ghidini, C. (2001). On the dimensions of context dependence: Partiality, approximation, and perspective. Proceedings of CONTEXT 2001.

Bradley, N. A., \& Dunlop, M. D. (2003). Towards a multidisciplinary user-centric design framework for context-aware applications. Proceedings of the 1 st UK-UbiNet Workshop.

Brezillon, P., \& Abu-Hakima, S. (1995). Using the knowledge in its context: Report of the IJCAI '93 Workshop. AI Magazine, 16, 87-91.

Brown, P. J. (1996). The Stick-e document: A framework for creating context-aware applications. Proceedings of EP '96.

Brown, P.J. (2000). Research issues in context-aware retrieval: The weather. Unpublished report, Department of Computer Science, University of Exeter, Exeter, England.

Brown, P. J., Bovey, J. D., \& Chen, X. (1997). Context-aware applications: From the laboratory to the marketplace. IEEE Personal Communications, 4(5), 58-64.

Bunt, H. (1997). Context and dialogue control. Proceedings of CONTEXT' 97. 
Burrell, J., \& Gay, G. K. (2001). Collectively defining context in a mobile, networked computing environment. Short talk in CHI Extended Abstracts, May 2001.

Chambers 21st century dictionary. (1996). Edinburgh, Scotland: Chambers.

Chen, G., \& Kotz, D. (2000). A survey of context-aware mobile computing research. Technical Report TR2000-381, Department of Computer Science, Dartmouth College.

Cheverst, K., Davies, N., Mitchell, K., Friday, A., \& Efstratiou, C. (2000). Developing a context-aware electronic tourist guide: Some issues and experiences. Proceedings of CHI 2000.

Connolly, J. H. (2001). Context in the study of human languages and computer programming languages: A comparison. Proceedings of CONTEXT 2001.

Coutaz, J., \& Rey, G. (2002). Recovering foundations for a theory of contextors. Presentation delivered at the 4 th International Conference on Computer-Aided Design of User Interfaces.

Davies, G. M., \& Thomson, D. M. (1988). Introduction. In G. M. Davies \& D. M. Thomson (Eds.), Memory in context; context in memory (pp. 1-10). Chichester, England: Wiley.

Dervin, B. (1997). Given a context by any other name: Methodological tools for taming the unruly beast. In B. Dervin \& L. Foreman-Wernet (with E. Lauterbach) (Eds.), Sense-making methodology reader: Selected writings of Brenda Dervin (pp. 111-132). Cresskill, NJ: Hampton.

Dewey, J. (1960). Context and thought. In R. Bernstein (Ed.), John Dewey: On experience, nature and freedom: Representative selections (pp. 88-110). New York: Bobbs-Merrill.

Dey, A. K. (1998). Context-aware computing: The CyberDesk Project. Proceedings of AAAI 1998 Spring Symposium Series on Intelligent Environments.

Dey, A. K. (2001, September). Supporting the construction of context-aware applications. Presentation delivered at the Dagstuhl Seminar on Ubiquitous Computing.

Dey, A. K. \& Abowd, G. D. (1999). Towards a better understanding of context and context-awareness. Technical Report GIT-GVU-99-22, Georgia Institute of Technology.

Dey, A. K., Abowd, G. D., \& Wood, A. (1999). CyberDesk: A framework for providing self-integrating context-aware services. Knowledge-Based Systems, 11, 3-13.

Dey, A. K., Salber, D., \& Abowd, G. D. (2001). A conceptual framework and a toolkit for supporting the rapid prototyping of context-aware applications. Human-Computer Interaction, 16, 97-166.

Dourish, P. (2001). Seeking a foundation for context-aware computing. Human-Computer Interaction, 16, $\mathrm{xxx}-\mathrm{xxx}$.

Ekbia, H. R., \& Maguitman, A. G. (2001). Context and relevance: A pragmatic approach. Proceedings of CONTEXT 2001.

Fetzer, A. (1997). Recontextualizing context. Proceedings of Context Organiser workshop at ECCS' 97.

Funk, H. B., \& Miller, C. A. (1997). Context sensitive interface design. Proceedings of CONTEXT' 97.

Helal, A. S., Moore, S. E., \& Ramachandran, B. (2001). Drishti: An integrated navigation system for visually impaired and disabled. Proceedings of the 5th International Symposium on Wearable Computers. 
Huang, Q. (2002). Supporting context-aware computing in ad hoc mobile environments. Technical Report WUCS-02-36, Department of Computer Science and Engineering, Washington University.

Kim, J., Yae, S., \& Ramakrishna, R. S. (2001). Context-aware application framework based on open service gateway. Proceedings of the International Conference on Info-Tech and Info-Net.

Kokinov, B., \& Grinberg, M. (2001). Simulating context effects in problem solving with AMBR. Proceedings of CONTEXT 2001.

Matsui, T. (2001). Experimental pragmatics: Towards testing relevance-based predictions about anaphoric bridging inferences. Proceedings of CONTEXT 2001.

Moran, T., \& Dourish, P. (Eds.). (2001). Introduction to context-aware computing [Special issue]. Human-Computer Interaction, 16.

Mynatt, E., Essa, I., \& Rogers, W. (2000). Increasing the opportunities for aging-in-place. Proceedings of the ACM Conference on Universal Usability. New York: ACM.

Ochs, E. (1979). What a child language can contribute to pragmatics. In E. Ochs \& B. B. Schriffin (Eds.), Developmental pragmatics (pp. 1-17). New York Academic.

Oxford pocket dictionary of current English. (1992). Oxford, England: Clarendon.

Pashtan, A., Blattler, R., Heusser, A., \& Scheuermann, P. (2003). CATIS: A Context-Aware Tourist Information System. Proceedings of the 4th International Workshop of Mobile Computing.

Preece, J., Rogers, Y., Sharp, H., Benyon, D., Holland, S., \& Carey, T. (1994). Human-computer interaction. Harlow, England: Addison-Wesley.

Ryan, N., Pascoe, J., \& Morse, D. (1997). Enhanced reality fieldwork: The context-aware archaeological assistant. In V. Gaffney, M. Van Leusen, \& S. Exxon (Eds.), Computer applications in archaeology (pp. 34-45).

Salber, D., Siewiorek, D. P., \& Smailagic, A. (2001). Supporting mobile workgroups on a wireless campus. Proceedings of Mobile HCI 2001.

Schilit, B., Adams, N. \& Want, R. (1994). Context-aware computing applications. Proceedings of the 1st International Workshop on Mobile Computing Systems and Applications.

Schilit, B., \& Theimer, M. (1994). Disseminating active map information to mobile hosts. IEEE Network, 8(5), 22-32.

Schmidt, A. (2001). Context-awareness, disappearing and distributed user interfaces: Experience, open issues and research questions. Presentation delivered at the Dagstuhl Seminar on Ubiquitous Computing.

Selker, T., \& Burleson, W. (2000). Context-aware design and interaction in computer systems. IBM Systems Journal, 39, 880-891.

Smith, S. (1988). Environmental context-dependent memory. In G. M. Davies \& D. M. Thomson (Eds.), Memory in context; context in memory (pp. 13-34). Chichester, England: Wiley.

Theodorakis, M., \& Spyratos, N. (2002). Context in artificial intelligence and information modeling. Proceedings of SETN 2002.

Tijus, C. (2001). Contextual categorization and cognitive phenomena. Proceedings of CONTEXT 2001.

Unwired Express. (2002). What is cntext? Retrieved Month, Day, Year, from http://www.unwiredexpress.com 
Ward, A., Jones, A., \& Hopper, A. (1997). A new location technique for the active office. IEEE Personal Communications, 4(5), 42-47.

Wittgenstein, L. (1958). Philosophical investigations. Oxford, England: Blackwell.

Zetie, C. (2002a). Context: Delivering the true value of enterprise mobile computing. Retrieved Month, Day, Year, from http://www.unwiredexpress.com/products/ downloads/ContextWPv2.pdf

Zetie, C. (2002b). Market overview-The emerging context-aware software market. Retrieved Month, Day, Year, from http://www.unwiredexpress.com/products/downloads/ context_market_overview.pdf

Ziemke, T. (1997). Embodiment of context. Proceedings of ECCS. 